\section{JTI}

JOURNAL OF

TRAUMA AND INJURY

\title{
Minimally Invasive Stabilization with Percutaneous Screws Fixation of APC-3 Pelvic Ring Injury
}

\author{
Beom-Soo Kim, M.D. ${ }^{1}$, Jong-Keon Oh, M.D. ${ }^{1}$ Jae-Woo Cho, M.D. ${ }^{1}$, \\ Do-Hyun Yeo, M.D. ${ }^{1}$, Jun-Min Cho, M.D. ${ }^{2}$ \\ Departments of ${ }^{1}$ Orthopedic Surgery, ${ }^{2}$ General Surgery, Korea University Guro Hospital, \\ Seoul, Korea
}

Received: May 31, 2018

Revised: August 29, 2018

Accepted: September 3, 2018

\section{Correspondence to}

Jun-Min Cho, M.D.

Department of General Surgery, Korea University Guro Hospital, 148 Guro-

dong-ro, Guro-gu, Seoul 08308, Korea

Tel: $+82-2-2626-1114$

Fax: +82-2-2626-2024

E-mail: nobleedings@gmail.com

Pelvic fractures are associated with life-threatening injuries and high rates of morbidity and mortality. Management of internal blood loss associated with unstable pelvic ring injuries is paramount during the initial period. The reconstruction of the pelvic ring is of importance because it is a major contributor to the stability of the pelvic ring. We report the case of a 25-year-old man who had an unstable pelvic ring fracture combined with rupture of an obturator artery and had a successful and satisfactory treatment using minimally invasive surgery with percutaneous antegrade screw fixation.

Keywords: Pelvis; Fracture; Osteosynthesis; Minimally invasive surgical procedure; Therapeutic embolization

\section{INTRODUCTION}

Pelvic ring fractures represent only $3 \%$ of all fractures, but typically arise from high-energy trauma and have a high incidence of associated injuries that lead to significant morbidity and mortality [1,2]. Initial management of hemodynamically unstable pelvic ring injuries is paramount [3]. In addition to general measures of circulatory stabilization, reconstruction of the pelvic anatomy and restoration of the stable and non-expandable compartment are the goals of patient care [4]. Especially, the reconstruction of the posterior pelvic ring is of great importance because it is a major contributor to the stability of the pelvic ring [1]. In addition, biomechanical studies have also shown that additional osteosynthesis of anterior lesions significantly improves stability [5].

We report the case of a 25-year-old man who had an unstable pelvic ring fracture 
with sacral dysmorphism combined with rupture of an obturator artery. The patient treated with minimally invasive surgery using the plate and percutaneous antegrade screw showed a successful and satisfactory outcome.

\section{CASE REPORT}

A 25-year-old man arrived at our emergency department 18 minutes after a traffic accident during riding a motorcycle. His vital signs were stable with a blood pressure of $110 / 70 \mathrm{~mm} \mathrm{Hg}$ and pulse rate of $80 / \mathrm{min}$ at the time of arrival. He complained of pain in the bilateral pelvic region, left forearm, right knee, and face. The motor and sensory abilities were intact and blood circulation was palpable in both of his upper and lower extremities. After 1 hour from the time of arrival, his blood pressure was 100/60 $\mathrm{mmHg}$ and pulse rate was $80 / \mathrm{min}$, and radiographs and computed tomography (CT) scan were performed. When the patient was brought back to the emergency department from the radiological department, his systolic blood pressure had decreased to $60 \mathrm{mmHg}$, and the radiographs and CT scan showed an anterior posterior compression (APC) type 3 pelvic ring injury with a $6.2 \mathrm{~cm}$ diastasis of the symphysis pubis and rami fractures (Fig. 1). An abdominal pelvic CT scan showed an implication of the finding of contrast extravasation around the obturator artery. The patient's vital signs became unstable, and two units each of packed red cells and fresh frozen plasma were immediately administered with a large amount of normal saline. With application of a pelvic binder, we used a portable radiographic device to confirm that the diastatic distance of the pubic symphysis was decreased. At 30 minutes after application of the pelvic binder, his vital signs were stabilized. To resolve the extravasation in the pelvic cavity, a transcatheter arterial embolization (TAE) was performed immediately. Bleeding in the left obturator artery territory was confirmed, and coil embolization using was performed (Fig. 2). After
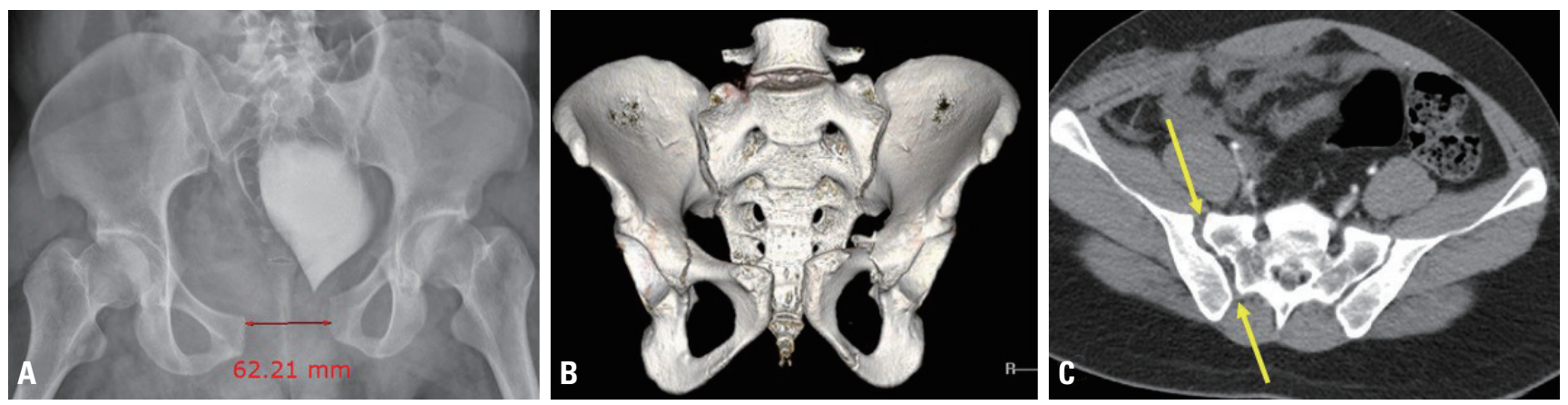

Fig. 1. (A, B) The radiograph and CT scans show an anterior posterior compression (APC) type 3 pelvic ring injury with a 6.2-cm diastasis of the symphysis pubis (red arrow) and fractures of the bilateral superior and inferior ramus area. (C) The axial CT image shows a widening of the sacroiliac joint presumed to be a disruption of the anterior and posterior sacroiliac ligament (yellow arrows). CT: computed tomography.
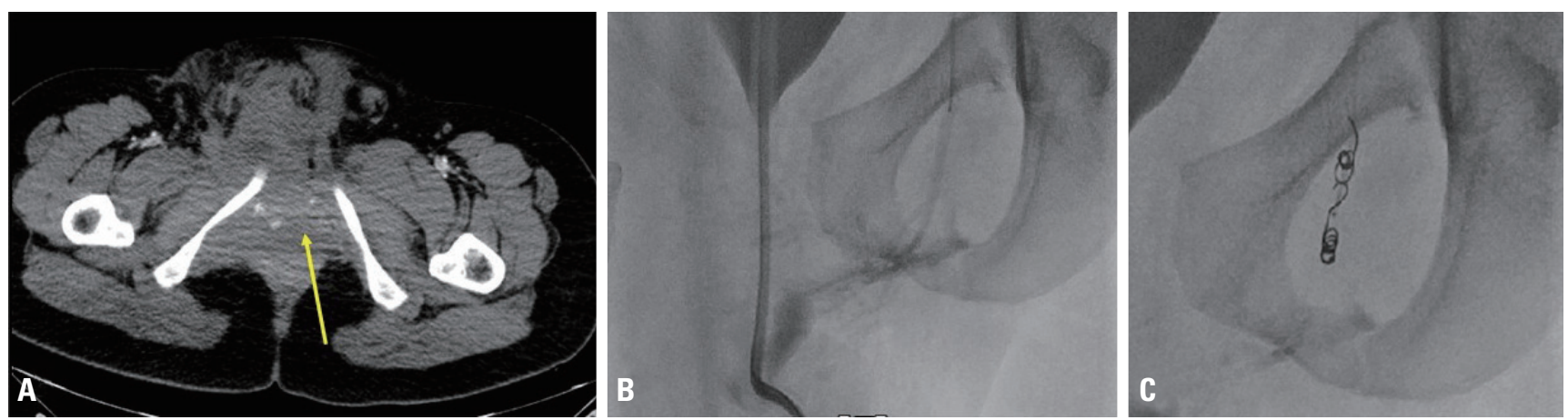

Fig. 2. (A) The abdominal pelvic CT scan shows an implication of the finding of contrast extravasation (yellow arrrow) around the left obturator artery. (B, C) The bleeding in the left obturator artery territory was confirmed, and coil embolization using coil was performed. CT: computed tomography. 


\section{JTI}

application of the pelvic binder and embolization, the vital signs of the patient were stabilized. After management of his general condition, 3 days after the time of arrival, the patient underwent pelvic fracture fixation.

The patient was placed in a supine position on a flat radiolucent table. His arms were placed on armrests in an abducted position to avoid interference with the $\mathrm{C}$-arm during inlet view. The entire procedure was performed using an intraoperative C-arm imaging system. The surgical site was prepared, and the bilateral hemipelvis, buttocks, and proximal thigh were exposed. After draping of the surgical site, U-drapes were placed around the surgical field and the area was prepped a final time. First, the anterior pelvic ring was stabilized using a reduction clamp through the Pfannenstiel approach to restorate the diastasis of the symphysis pubis. After confirming the location of the bilateral ramus fracture, we decided to perform antegrade percutaneous screw fixation first on the right ramus. After obtaining an anatomical reduction, a soft tissue sleeve was used to insert a guide wire passing from the supra-acetabular area to the superior pubic rami. Multiple fluoroscopic views were obtained to confirm the place of the inserted guide wire. A 7.0-mm-diameter, 16- $\mathrm{mm}$ partially threaded cannulated screw was then placed over the guide wire for compression of the fracture site. The screw was fixed in the same way to the left ramus fracture (Fig. 3).

After fixation of the bilateral screws through an incision made with the Pfannenstiel approach, an anterior plate was fixed using a six-hole single unlocked $3.5-\mathrm{mm}$ plate (Synthes Inc., West Chester, PA, USA). The plate screws were placed to maximize the osseous purchase to avoid an
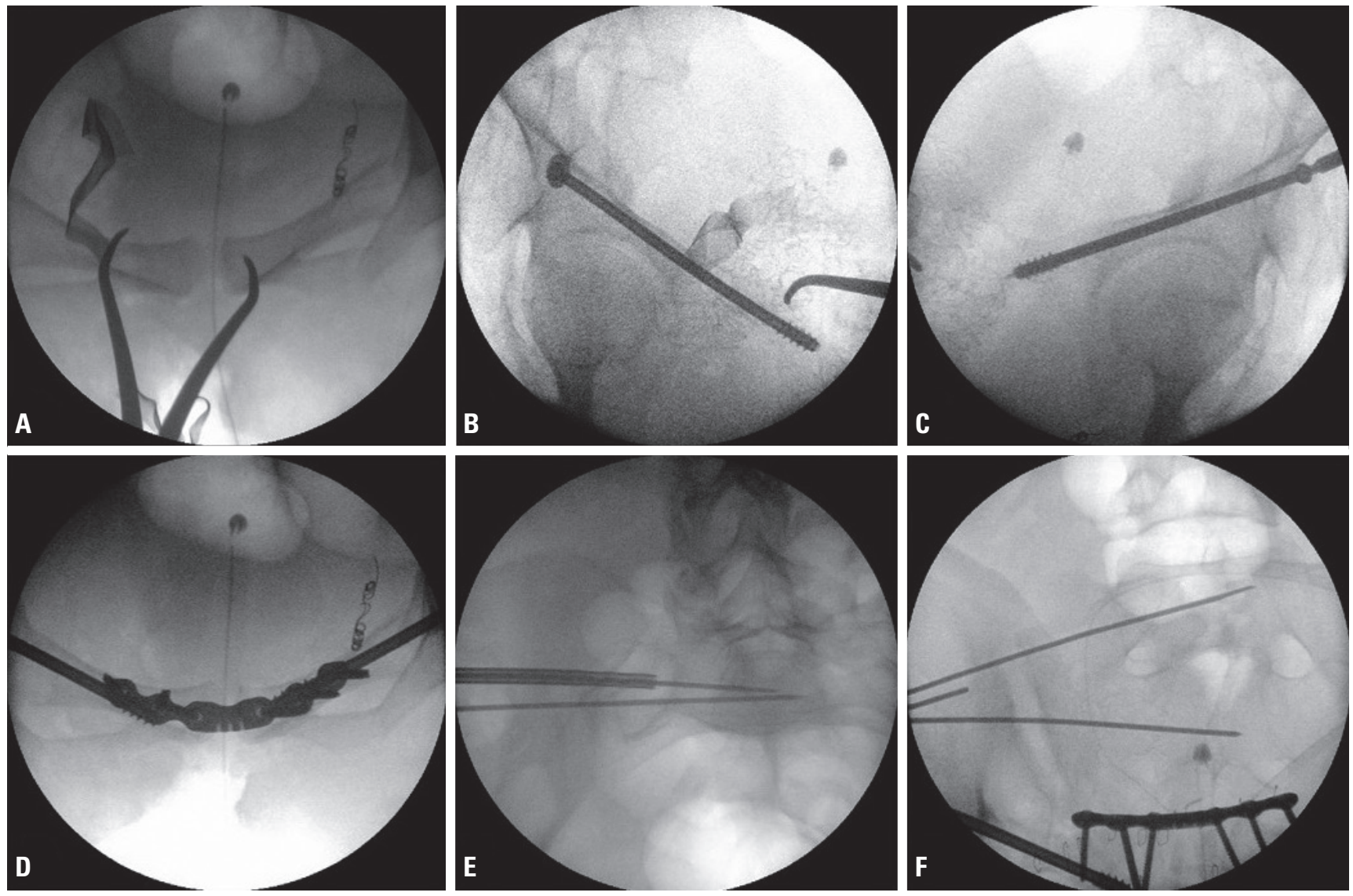

Fig. 3. The entire procedure was performed using an intraoperative C-arm imaging system. (A) The anterior pelvic ring was stabilized using a reduction clamp to resolve the diastasis of the symphysis pubis. (B, C) A 7.0-mm-diameter, 16-mm partially threaded cannulated screw was placed over the guide wire for compression of the bilateral and lateral ramus fracture sites. (D) After fixation of the bilateral screws, through an incision with the Pfannenstiel approach, an anterior plate was fixed using a six-hole single unlocked 3.5-mm plate. (E) The inlet view shows two sacroiliac guide wires placed in a proper position at the bodies of S1 and S2. (F) The outlet view shows the sacroiliac guide wire placed in a slightly oblique direction because of sacral dysmorphism. 
extraosseous location. After fixation of the anterior plate, the displaced right sacroiliac (SI) joint was fixed to stabi- lize the posterior pelvic ring. As pelvic dysmorphism was found in the preoperative planning, percutaneous screw
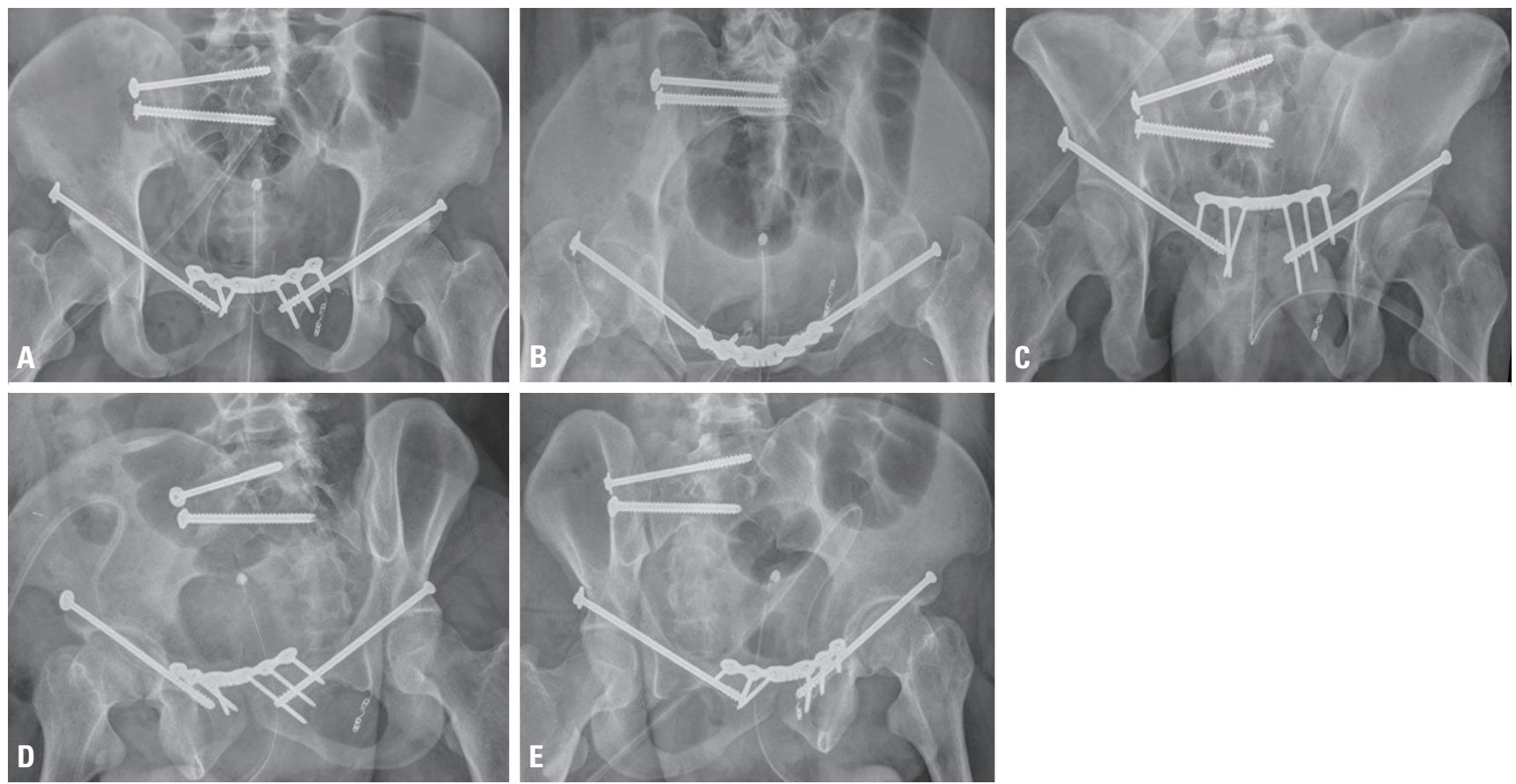

Fig. 4. The postoperative radiographs demonstrate an anatomic reduction with plate and screws in the anteroposterior (A), inlet (B), outlet (C), and oblique views $(\mathrm{D}, \mathrm{E})$.
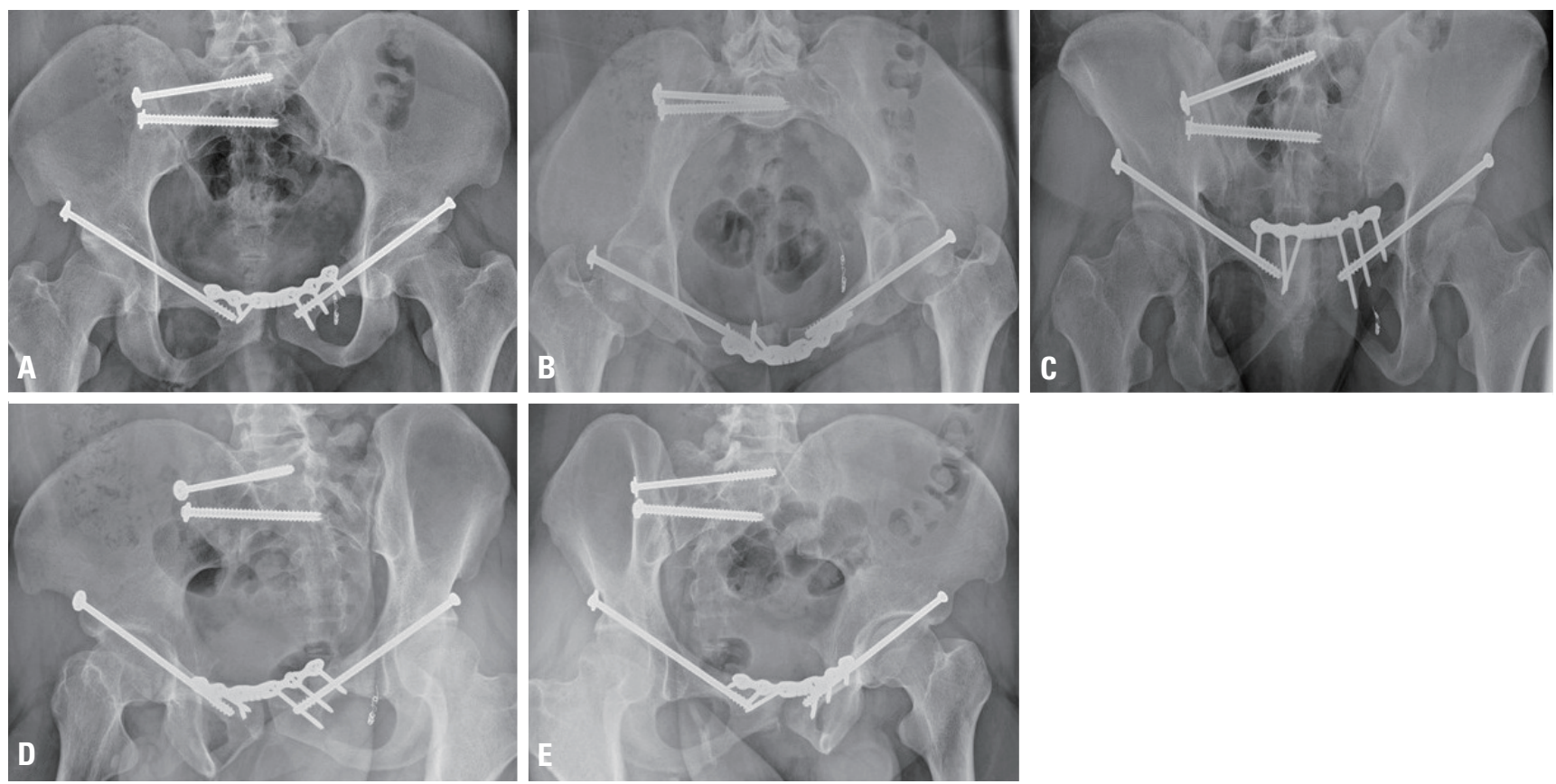

Fig. 5. The follow-up radiographs at 1 year 6 months after index surgery demonstrate complete bony healing in the anteroposterior (A), inlet (B), outlet (C), and oblique views $(D, E)$. 
fixation was performed in different directions at the S1 and S2 levels. First, a guide pin was inserted in the correct position at the S2 level, and at the S1 level, only the guide pin was inserted in the oblique direction to the S1 sacral body (Fig. 3). For compression of the SI joint, a partially threaded screw was used for S1, and then a full threaded screw was used for insertion of a screw in S2 (Fig. 4).

After the operation, compression stocking and pneumatic compression devices to prevent deep vein thrombosis were used for 10 days prophylactically. Physical therapy was begun on the first postoperative day, and the patient was instructed to ambulate with partial weight bearing with the aid of a walker. The patient was instructed to start full weight bearing 6 weeks after the operation. The radiographs at the 18th month of the postoperative period showed no evidence of degenerative signs, and the clinical outcome was good (Fig. 5).

\section{DISCUSSION}

The management of patients with pelvic ring injuries is challenging and requires several disciplines. The clinical condition of the patient can rapidly decline; hence, surgeons must be aware of all the important considerations when administering the initial management of these patients [6]. Hypovolemic shock continues to be a major contributing factor to the mortality after pelvic ring injuries [4]. Sources of bleeding include the intra-pelvic venous plexus, disrupted arteries, and cancellous bone or combinations of all the above [7]. In this case, the blood pressure suddenly decreased with a systolic blood pressure of $60 \mathrm{mmHg}$. We considered that several combined factors caused the low blood pressure and shock in this patient.

APC injuries are subdivided into types 1, 2, and 3, and correlate with relative stability [6]. In this case, diastasis of the symphysis pubis was $6.2 \mathrm{~cm}$, which determined the type of APC 3 injury, with a symphyseal diastasis of $>2.5$ $\mathrm{cm}$ and widened SI joints due to disruption of the sacrospinous and anterior and posterior SI ligaments. Severe APC injuries create a complete diastasis of the anterior pelvis, and partial or complete diastasis of the posterior pelvis [8]. The tensile and shear forces to the pelvic vas- culature result in greater disruption of the vessels and more bleeding than in other injury mechanisms $[1,4]$. In this case, a pelvic binder was provided immediately after the unstable pelvic ring fracture was confirmed using radiological images, and urgent TAE was performed using angiography in cooperation with the intervention team of the radiological department to successfully block the bleeding obturator artery. In previous studies, different algorithms have been proposed for the management of unstable pelvic fractures $[3,4,9,10]$. If a vital sign of patient is hemodynamically unstable, management determinations are influenced by the availability of resources such as a surgical team instantaneously available with a surgeon familiar with the principles of damage control and preperitoneal packing and/or an angiography suite as well as with a radiologist for an urgent embolization. Our institution is also treating the patient with an algorithm similar with that used in the previous studies.

The surgical treatment of pelvic and acetabular fractures is technically demanding [1,8,11-13]. The aim is to achieve anatomical or near-anatomical restoration of the articular surface and accurate reduction of the pelvic ring in mechanically unstable injuries. In general, if only diastasis of the symphysis pubis exists in the APC-type pelvic ring injury, only anterior plating can be appropriate treatment if it is indicated. However, an extended approach such as the ilioinguinal or modified Stoppa approach extended beyond the Pfannenstiel approach is needed to fix the ramus fracture, which is in close proximity to the acetabular area, to stabilize the pelvic ring [14]. The extended approach has disadvantages in that the length of the incision is enlarged more than the simple anterior approach such as the Stoppa and Pfannenstiel approaches, the operation time is long, and the possibilities of major blood loss and postoperative infection are increased $[5,15,16]$. Percutaneous pelvic fixation has been receiving more attention to avoid extensile surgical approaches. In this case, a minimally invasive surgery was decided with measures to minimize bleeding in consideration of his general condition and reduce the operation time to improve the prognosis of the patient, and successful fracture reduction and fixation were achieved.

Understanding the dysmorphism of the sacrum was essential for stabilizing the SI joint of the patient. Dys- 
morphic osseous characteristics include collinearity of the upper portion of the sacrum and iliac crests on the outlet radiographic view and the presence of mammillary bodies at the sacral ala, noncircular anterior sacral foramina, residual upper sacral disks, a tongue-in-groove sacroiliac joint surface, and cortical indentation of the ala on the inlet radiograph [17]. In addition, these patients have an acute alar slope as seen on the lateral view of the sacrum. In this case, most of the above-mentioned features of sacral dysmorphism appeared on the radiograph and CT scans. A special screw fixation method at the S1 level is required for characteristic reasons that the horizontal screws placed across a dysmorphic sacrum will result in iatrogenic nerve injury. In this case, understanding of this sacral dysmorphism and correct fixation of the screw to the narrow and oblique safe zone of the upper sacrum increased the fixation force and compression of the SI joint.

Limitation of this case, a pelvic ring injury of APC type was detected on radiography when the blood pressure decreased suddenly, and a pelvic sheet was applied urgently. If a pelvic sheet or pelvic binder was administered immediately before blood pressure dropped, hemodynamically instability might not have occurred. However, Successful stabilization of vital signs was attained by active and quick cooperation on the extravasation of the artery on the CT scan. We performed an appropriate treatment with a minimally invasive surgical technique with careful preoperative planning for an APC-3 pelvic ring injury with sacral dysmorphism. The treatment using separated antegrade rami screws fixation and anterior symphyseal plating is useful to stabilization of unstable pelvic ring injury.

\section{REFERENCES}

1. Tonetti J. Management of recent unstable fractures of the pelvic ring. An update conference supported by the Club Bassin Cotyle. (Pelvis-Acetabulum Club). Orthop Traumatol Surg Res 2013;99(1 Suppl):S77-86.

2. Matta JM, Tornetta P 3rd. Internal fixation of unstable pelvic ring injuries. Clin Orthop Relat Res 1996;(329):129-40.

3. Lindahl J, Hirvensalo E. Outcome of operatively treated type-C injuries of the pelvic ring. Acta Orthop 2005;765:667-78.

4. Gruen GS, Leit ME, Gruen RJ, Peitzman AB. The acute manage- ment of hemodynamically unstable multiple trauma patients with pelvic ring fractures. J Trauma 1994;36:706-11; discussion 711-3.

5. Routt MC Jr, Nork SE, Mills WJ. Percutaneous fixation of pelvic ring disruptions. Clin Orthop Relat Res 2000;375:15-29.

6. Richard MJ, Tornetta P 3rd. Emergent management of APC-2 pelvic ring injuries with an anteriorly placed C-clamp. J Orthop Trauma 2009;23:322-6.

7. Pohlemann T, Gänsslen A, Schellwald O, Culemann U, Tscherne H. Outcome after pelvic ring injuries. Injury 1996;27 Suppl 2:B31-8.

8. Gu R, Huang W, Yang L, Liu H, Xie K, Huang Z. Comparisons of front plate, percutaneous sacroiliac screws, and sacroiliac anterior papilionaceous plate in fixation of unstable pelvic fractures. Medicine (Baltimore) 2017;96:e7775.

9. Karadimas EJ, Nicolson T, Kakagia DD, Matthews SJ, Richards PJ, Giannoudis PV. Angiographic embolisation of pelvic ring injuries. Treatment algorithm and review of the literature. Int Orthop 2011;35:1381-90.

10. Tötterman A, Dormagen JB, Madsen JE, Kløw NE, Skaga NO, Røise O. A protocol for angiographic embolization in exsanguinating pelvic trauma: a report on 31 patients. Acta Orthop 2006;77:462-8.

11. Giannoudis PV, Tzioupis CC, Pape HC, Roberts CS. Percutaneous fixation of the pelvic ring: an update. J Bone Joint Surg Br 2007;89:145-54.

12. Dahill M, McArthur J, Roberts GL, Acharya MR, Ward AJ, Chesser TJS. The use of an anterior pelvic internal fixator to treat disruptions of the anterior pelvic ring: a report of technique, indications and complications. Bone Joint J 2017;99B:1232-6.

13. Choy WS, Kim KJ, Lee SK, Park HJ. Anterior pelvic plating and sacroiliac joint fixation in unstable pelvic ring injuries. Yonsei Med J 2012;53:422-6.

14. Guthrie HC, Owens RW, Bircher MD. Fractures of the pelvis. J Bone Joint Surg Br 2010;92:1481-8.

15. Ceylan H, Selek O, Sarlak AY. Percutaneous fixation of anterior column acetabular fracture in a renal transplant recipient. Case Rep Orthop 2013;2013:842390.

16. Tempelaere C, Vincent C, Court C. Percutaneous posterior fixation for unstable pelvic ring fractures. Orthop Traumatol Surg Res 2017;103:1169-71.

17. Miller AN, Routt ML Jr. Variations in sacral morphology and implications for iliosacral screw fixation. J Am Acad Orthop Surg 2012;20:8-16. 